\title{
Neuropathology of human Alzheimer disease after immunization with amyloid- $\beta$ peptide: a case report
}

\author{
James A.R. Nicoll ${ }^{1,2}$, David Wilkinson ${ }^{1,3}$, Clive Holmes ${ }^{1,3}$, Phil STearT $^{2}$, \\ HANNAH MARKHAM ${ }^{1,2} \&$ ROY O. WelleR ${ }^{1,2}$ \\ ${ }^{1}$ Division of Clinical Neurosciences, University of Southampton, Southampton, UK \\ ${ }^{2}$ Neuropathology, Department of Pathology, Southampton General Hospital, Southampton, UK \\ ${ }^{3}$ Memory Assessment and Research Centre, Moorgreen Hospital, Southampton, UK \\ Correspondence should be addressed to J.A.R.N.; e-mail: J.Nicoll@soton.ac.uk
}

Published online 17 March 2003; doi:10.1038/nm840

\begin{abstract}
Amyloid- $\beta$ peptide $(A \beta)$ has a key role in the pathogenesis of Alzheimer disease (AD). Immunization with $A \beta$ in a transgenic mouse model of $A D$ reduces both age-related accumulation of $A \beta$ in the brain ${ }^{1}$ and associated cognitive impairment ${ }^{2,3}$. Here we present the first analysis of human neuropathology after immunization with $A \beta$ (AN-1792). Comparison with unimmunized cases of $\mathrm{AD}(n=7)$ revealed the following unusual features in the immunized case, despite diagnostic neuropathological features of AD: (i) there were extensive areas of neocortex with very few $A \beta$ plaques; (ii) those areas of cortex that were devoid of $A \beta$ plaques contained densities of tangles, neuropil threads and cerebral amyloid angiopathy (CAA) similar to unimmunized $A D$, but lacked plaque-associated dystrophic neurites and astrocyte clusters; (iii) in some regions devoid of plaques, A $\beta$-immunoreactivity was associated with microglia; (iv) T-lymphocyte meningoencephalitis was present; and (v) cerebral white matter showed infiltration by macrophages. Findings (i)-(iii) strongly resemble the changes seen after $A \beta$ immunotherapy in mouse models of $A D^{1-6}$ and suggest that the immune response generated against the peptide elicited clearance of $A \beta$ plaques in this patient. The T-lymphocyte meningoencephalitis is likely to correspond to the side effect seen in some other patients who received AN-1792 (refs. 7-9).
\end{abstract}

A 72-year-old woman with a 5-year history of gradually progressive memory impairment presented with worsening confusion and disorientation. Her Mini Mental State Examination (MMSE) score $(23 / 30)$ represented a three-point deterioration in two years. She had global cognitive impairment and satisfied the National Institute of Neurological and Communicative Disorders and Stroke-Alzheimer's Disease and Related Disorders Association's criteria for probable AD, with no cardiovascular risk factors and a modified Haschinski score $<4$. Therapy with rivastigmine tartrate, a cholinesterase inhibitor, resulted in improvements in the Alzheimer's Disease Assessment Scale cognitive section (ADAS cog), MMSE, clock drawing and verbal fluency, but ten months later she had returned to baseline levels on all these parameters. The patient was then enrolled in a randomized, double-blind, multiple-dose immunogenicity study of A 42 (AN-1792; Elan Pharmaceuticals). She received her first injection, containing $50 \mu \mathrm{g}$ of AN-1792, in July 2000. This was repeated 4, 12 and 24 weeks later with no apparent adverse effects. A fifth injection with a reformulated preparation containing polysorbate-80, subsequently used in a multinational phase $2 \mathrm{a}$ trial, was given 36 weeks after the first injection. Four weeks after her last injection, her cognitive test results were unchanged (MMSE 23), but at six weeks she suddenly became unwell with dizzy spells, drowsiness, an unstable gait and fever. Two weeks after that, she deteriorated such that an MMSE could not be performed. Neuroimaging (Fig. 1a) showed extensive bilateral alterations in the cerebral white matter and enhancement on the brain surface. There was mild hydrocephalus; an isodense mass was identified above the splenium of the corpus callosum on the right side. The appearances were interpreted as representing either edema, possibly associated with an inflammatory process, or an infiltrating primary brain tumor. Therapy with dexamethasone was started. The patient remained relatively unchanged until she died in February 2002 from a pulmonary embolism 20 months after the first injection and 12 months after the last injection.

Post-mortem examination of the patient's brain showed atrophy of the cerebral cortex and white matter, with focal whitematter softening and granular change associated with ventricular enlargement (Fig. $1 b$ ). There was no mass lesion corresponding to that identified earlier by imaging. Neuritic plaques, neurofibrillary tangles and neuropil threads were identified in the cerebral neocortex by modified Bielshowsky, thioflavine $S$, anti-tau, anti- $\beta$-APP (amyloid precursor protein) and anti-A $\beta$ staining, providing histological confirmation of the clinical diagnosis of $\mathrm{AD}$ according to standard diagnostic criteria (Consortium to Establish a Registry for Alzheimer's Disease (CERAD) 'definite' and Braak \& Braak stage V-VI) ${ }^{10}$.

Where plaques were present in the immunized case, for example in the medial frontal lobes (Fig. 1c), they were patchy in contrast with the relatively uniform distribution in the unimmunized $\mathrm{AD}$ brain (Fig. $1 e$ and $f$ ). $\mathrm{A} \beta$ plaques were absent or sparse, however, throughout much of the neocortex, including extensive areas of the parietal (Fig. 1d), temporal, frontal and occipital lobes. In contrast, $A \beta$ plaques were numerous in the basal ganglia and cerebellum, which is usually a feature of relatively advanced AD. A $\beta$ plaque density (Fig. $1 g$ ) and $\mathrm{A} \beta$ load (Fig. $1 h$ ) were quantified by computerized image analysis in the immunized case and unimmunized AD cases $(n=7)$ in three regions of the temporal neocortex and two regions of the frontal neocortex that are usually severely affected in $\mathrm{AD}$. In the medial frontal gyrus, the plaque density of the immunized case was 140 plaques $/ \mathrm{mm}^{2}$, well within the range of the unimmunized AD cases (median 190, range 25.4-298 plaques $/ \mathrm{mm}^{2}$ ). However, in the cingulate gyrus and the three regions of the temporal lobe, the mean plaque density in the immunized case was below 
$a$

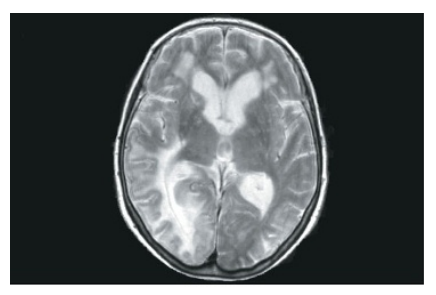

$b$

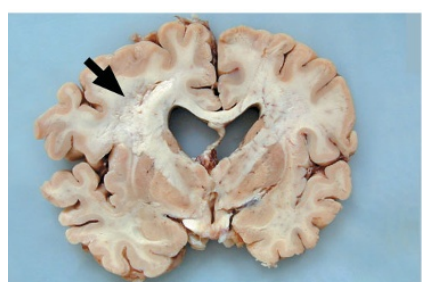

$e$

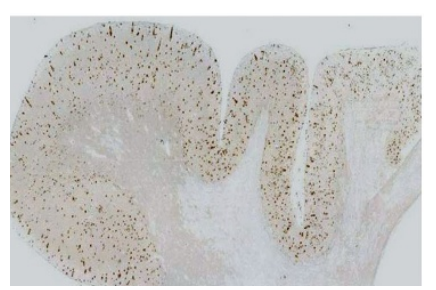

$f$

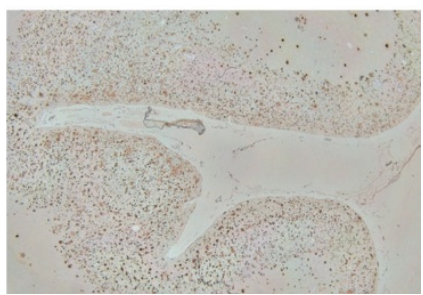

Fig. 1 Distribution and quantitation of $A \beta$ pathology. a, MRI scan at the time of acute illness, showing widespread signal alteration in cerebral white matter. $\boldsymbol{b}$, Corresponding granular change in cerebral white matter seen in a coronal slice of the cerebral hemispheres at post-mortem examination. $\boldsymbol{c}$, Patches of $A \beta$ plaques largely restricted to deep cortical laminae in the cingulate gyrus and medial frontal gyrus (immunized case). $\boldsymbol{d}$, Absence of $A \beta$ plaques from parietal neocortex of the immunized case, with persistent vascular amyloid (CAA). $\boldsymbol{e}$ and $\boldsymbol{f}$, Relatively uniform distribution of $A \beta$ plaques in corresponding regions of cerebral cortex in unimmunized AD. $\boldsymbol{g}$ and $\boldsymbol{h}$, Quantitative image analysis showing plaque density (plaques $/ \mathrm{mm}^{2} ; g$ ) and $\mathrm{A} \beta$ load $(h)$. For the immunized case in the medial frontal gyrus $(c)$, values were within the range of unimmunized Alzheimer's disease. In contrast, the plaque density and A $\beta$ load of the immunized case was lower than in unimmunized $A D$ in the cingulate gyrus, and the inferior, middle and superior temporal gyri. $\bullet$, immunized case; open shapes, 7 individual unimmunized AD cases.
C

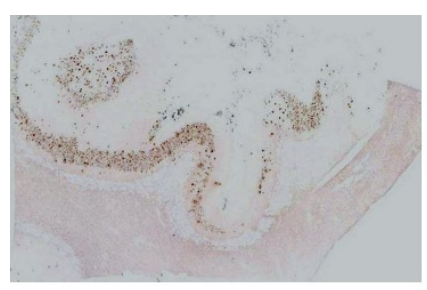

d

$g$

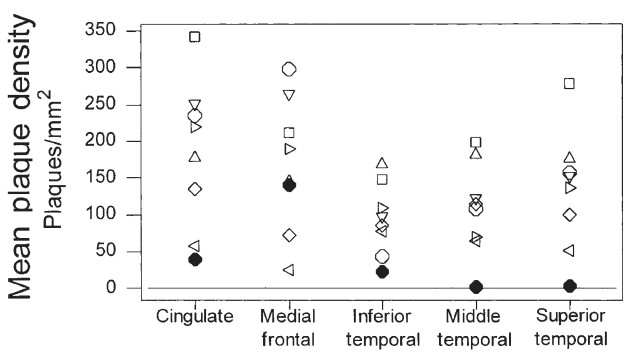

$h$

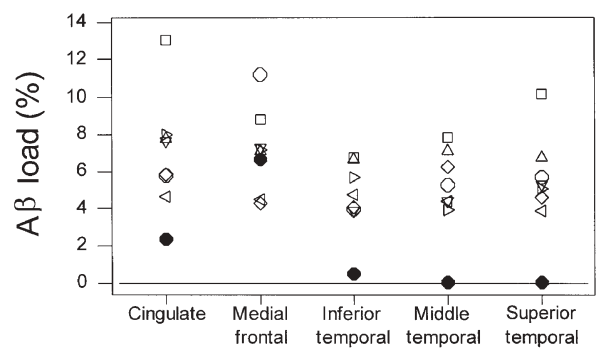

the range of the unimmunized $\mathrm{AD}$ cases, with very few plaques in the middle $\left(1.0\right.$ plaques $\left./ \mathrm{mm}^{2}\right)$ and superior temporal gyri (3.2 plaques $/ \mathrm{mm}^{2}$ ). Likewise, the mean $\mathrm{A} \beta$ load (percentage of microscope field immunostained for $\mathrm{A} \beta$; Fig. $1 \mathrm{~h}$ ) of the immunized case in the medial frontal gyrus (6.7\%) fell within the distribution of the load in the unimmunized AD cases (median $7.2 \%$, range $4.3-11.2 \%$ ), but was well below in the other four regions (cingulate $=2.4 \%$, inferior temporal $=0.46 \%$, middle temporal $=0.03 \%$, superior temporal $=0.04 \%$ ). Staining with thioflavine $S$ and Congo red was done (data not shown) to assess the possibility that the paucity of plaques detected by $A \beta$ immunohistochemistry in the immunized case was due to competition with the patient's own A $\beta$-specific antibodies. These amyloid stains showed plaque densities that corresponded to the $A \beta$ immunohistochemistry.

We assessed the distribution of other features of AD pathology in relation to this patchy distribution of $A \beta$ plaques in the immunized case by comparing the anatomical regions that had the highest (medial frontal gyrus) and the lowest (middle temporal gyrus) $A \beta$ loads (Table 1). Specific features associated with plaques in $\mathrm{AD}$ (such as clusters of tau-immunoreactive dystrophic neurites and clusters of glial fibrillary acid protein (GFAP)-immunoreactive astrocytes) were substantially less numerous in the middle temporal gyrus of the immunized case, corresponding with the paucity of $\mathrm{A} \beta$ plaques in that region compared with both the medial frontal gyrus in that case and the unimmunized AD cases. However, features of AD pathology that are not specifically associated with plaques (such as neurofibrillary tangles, neuropil threads and CAA, an accumulation of amyloid in the walls of blood vessels) were distributed relatively uniformly throughout the cerebral cortex in the immunized case, regardless of the variation in the density of $A \beta$ plaques (Fig. $2 a-d$ ). The intensity of IgG immunoreactivity of plaques did not differ between the immunized and unimmunized $\mathrm{AD}$ cases (Table 1 ).

Some of the neocortical areas devoid of $A \beta$ plaques contained small aggregates of granular or punctate $A \beta$ immunostaining (Fig. 2e), which corresponded closely in appearance and location to cells identified as phagocytic microglia immunoreactive for CD68 and human leukocyte antigen DR (Fig. $2 g$ ). This cellular pattern of $A \beta$ was observed with both $A \beta 40$ - and $A \beta 42$-specific antibodies (data not shown).

There was an infiltrate of lymphocytes in the leptomeninges (Fig. 3a-f), which was most dense in relation to amyloid-laden blood vessels. In addition, there was a sparse lymphocytic infiltrate in the cerebral cortex, in perivascular spaces, within the amyloid of the vessel walls, and within the parenchyma (Fig. $3 g$ and h). Immunohistochemistry identified the meningoencephalitis as being composed of $\mathrm{T}$ lymphocytes $\left(\mathrm{CD}^{+}\right.$and $\mathrm{CD}^{2} 5 \mathrm{RO}^{+}$; Fig. $3 b$ and $d$ ); the majority were $\mathrm{CD}^{+}$(Fig. $3 f$ and $h$ ) 
Table 1 Features of AD pathology in relation to $A \beta$ load

\begin{tabular}{|c|c|c|c|c|}
\hline \multirow[b]{3}{*}{$\mathrm{A} \beta$ load (\%) } & \multicolumn{2}{|c|}{ Medial frontal gyrus } & \multicolumn{2}{|c|}{ Middle temporal gyrus } \\
\hline & Unimmunized $^{\mathrm{a}}$ & Immunized & Unimmunized $^{\mathrm{a}}$ & Immunized \\
\hline & $7.2(4.3-11.2)$ & 6.7 & $5.3(3.9-7.8)$ & 0.03 \\
\hline Dystrophic neurite clusters (mean no. per $\times 10$ field) & $9.6(0.7-23.2)$ & 6.4 & $20.2(12.6-38.9)$ & 1.4 \\
\hline Astrocyte clusters (GFAP; mean no. per × 10 field) & $26.9(14.0-33.8)$ & 34.0 & $31.3(22.4-37.8)$ & 2.0 \\
\hline Plaque IgG immunoreactivity score & + (all cases) & + & $+(5$ cases $) ;++(2$ cases $)$ & $N A^{b}$ \\
\hline Neurofibrillary tangles (mean no. per $\times 10$ field) & $9.4(1.3-37.6)$ & 4.5 & $8.3(3.8-20.6)$ & 10.5 \\
\hline Neuropil thread score & $1.3(0-3)$ & 2 & $2.7(2-3)$ & 3 \\
\hline Cerebral amyloid angiopathy score & $1(0-3)$ & 3 & $1(0-3)$ & 3 \\
\hline
\end{tabular}

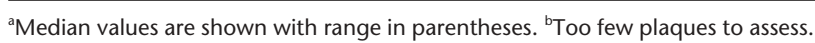

and very few were $\mathrm{CD}^{+}$(Fig. $3 e$ and $g$ ). B lymphocytes were not present (CD79a and CD20; Fig. 3c).

Meningoencephalitis is not a feature of AD pathology and is likely to be a consequence of the immunotherapy. Some of the other patients in the AN-1792 trial were found to have high cell counts in cerebrospinal fluid taken by lumbar puncture for investigation of adverse events with clinical features of meningoencephalitis ${ }^{9}$.

Corresponding with the magnetic resonance scans and macroscopic appearance of the brain, there were diffuse abnormalities affecting the cerebral white matter, with a marked reduction in the density of myelinated fibers (Fig. 3i) and extensive macrophage infiltration (Fig. 3j). Although its cause is unclear, this macrophage infiltration might have been responsible for the tumor-like appearance in the neuroimaging, particularly if the macrophage infiltration had been even more marked when the patient was alive. The macrophages in the white matter were not immunostained for $A \beta$, perhaps because they had metabolized previously phagocytosed $A \beta$ that was no longer immunoreactive, or because their presence was unrelated to phagocytosis of $A \beta$. Although depletion of myelinated fibers may be observed in $\mathrm{AD}$, particularly in cases with relatively severe CAA, it is not associated with macrophage infiltration. $a$

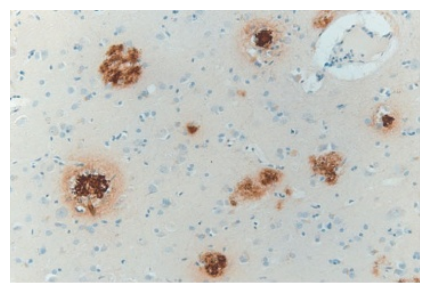

e

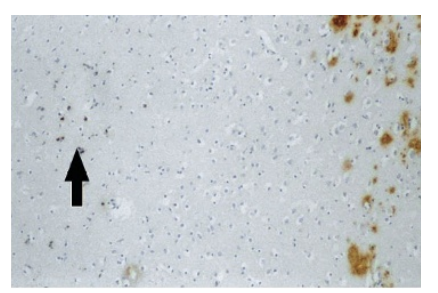

$b$

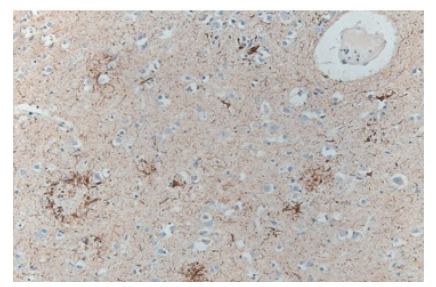

$f$

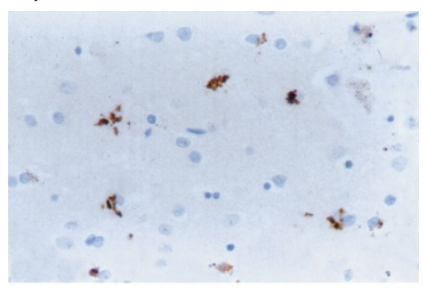

Fig. 2 Distribution of other features of $A D$ pathology in relation to $A \beta$ plaques and association of $A \beta$ with microglia. $\boldsymbol{a}-\boldsymbol{d}$, In the immunized case, cortical areas with abundant $A \beta$ plaques $(a)$ (corresponding sections immunostained for tau protein $(b)$ ) have plaque-associated dystrophic neurites, neurofibrillary tangles and neuropil threads similar to unimmunized AD. In contrast, cortical areas devoid of $A \beta$ plaques $(c)$ when immunostained for tau protein $(d)$ have neurofibrillary tangles and neuropil threads but lack clusters of dystrophic neurites. Persistence of vascular $A \beta(C A A)$ can be seen in area
Examination of the brain of our immunized patient showed features that are not normally seen in $\mathrm{AD}$ and that bear remarkable similarities to features of aged PDAPP mice, which express a mutant $A \beta$ precursor protein and normally accumulate $A \beta$ deposits, after $A \beta$ immunotherapy ${ }^{1-6}$. Both have a low density of $A \beta$ plaques in extensive areas of the cerebral cortex. In addition, both have a similar localization of $A \beta$ to microglia ${ }^{1}$. Fc-mediated phagocytosis of $A \beta$ by microglia in the presence of $A \beta$-specific antibodies was reported in an ex vivo study of plaque-laden tissue from both PDAPP mice and human $\mathrm{AD}^{5}$. If plaques were indeed cleared in this patient after immunization with $A \beta$ peptide, then it is possible that the low levels of antibody detected (positive titers of $1: 50$ at the time of the fifth injection, rising to $1: 1,004$ two weeks later and falling to 1:799 after four weeks) may be sufficient to effect plaque clearance over an extended period of time. Decoration of plaques by IgG and C 3 complement ${ }^{1}$ is a feature of the immune response that occurs in immunized PDAPP mice and that was seen in our immunized patient (data not shown). It is unclear whether this is a response to immunization, as similar intensities of IgG immunoreactivity were associated with plaques in the unimmunized AD cases (Table 1). Some differences may be anticipated between studies of immunized mice and humans because of the different timescales involved.

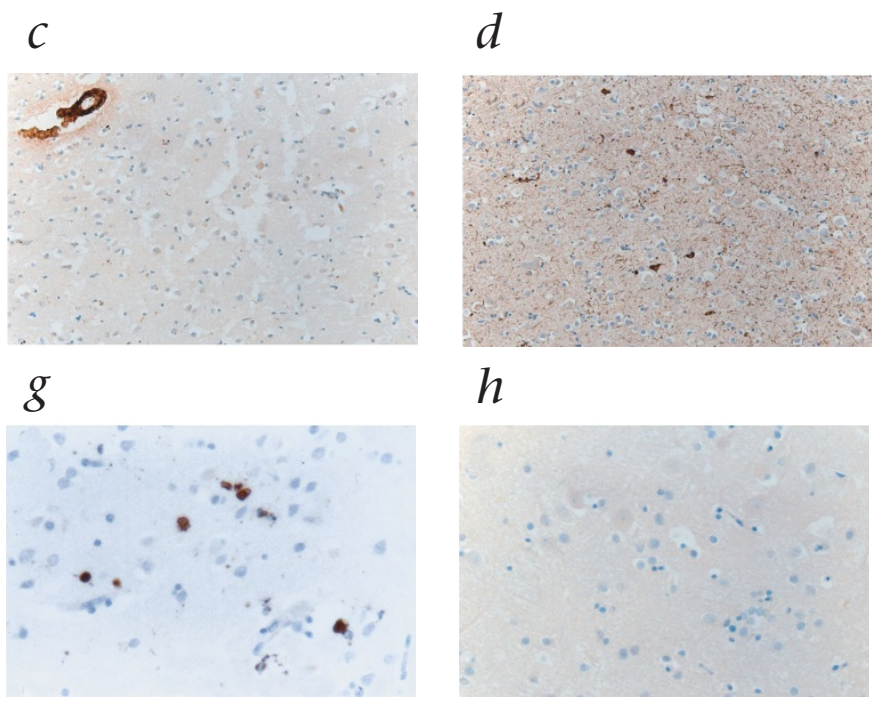

devoid of $A \beta$ plaques ( $c$; top left). $\boldsymbol{e}$, Interface between plaque-bearing cortex (right) and area devoid of plaques (left), showing unusual punctate immunoreactivity for $A \beta$ (arrow). $\boldsymbol{f}$ and $\boldsymbol{g}$, Higher-powered view of an area of parietal neocortex devoid of plaques $(f)$. This area shows punctate immunoreactivity for $A \beta$, which takes the form of clusters of granules and appears very similar to an adjacent section $(g)$ immunostained with an antibody against lysosomes of phagocytic microglia (CD68). $\boldsymbol{h}$, Negative control for the corresponding area, without primary antibody. 
$a$

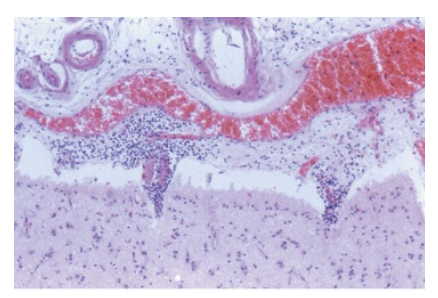

$e$

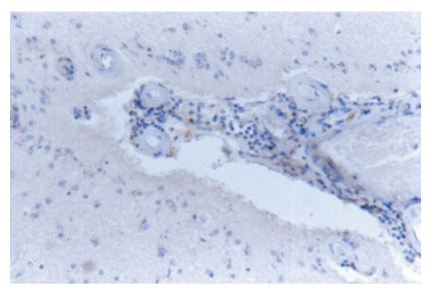

$b$

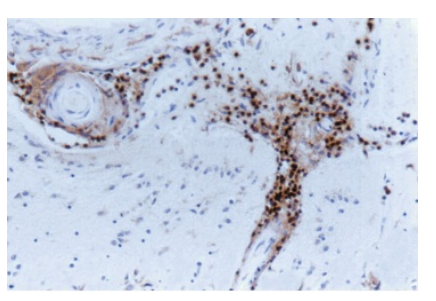

$f$

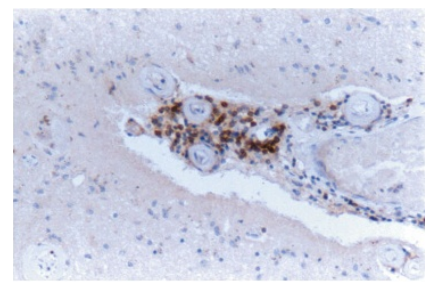

c

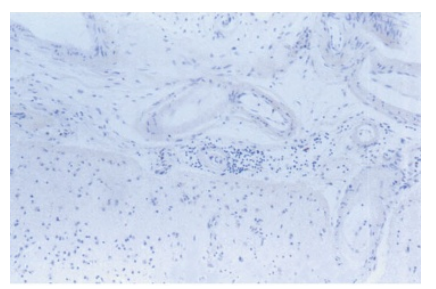

$g$

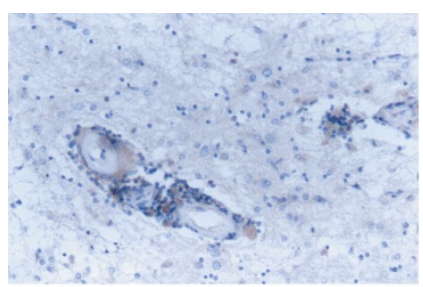

$i$

macrophages. $\boldsymbol{a}-\boldsymbol{h}$, Leptomeningeal infiltrate of lymphocytes stained with $\mathrm{H} \& \mathrm{E}(a)$ and immunostained with a T-lymphocyte antibody (CD3; $b$ ). No B lymphocytes were detected (CD79a; $)$. $d-h$, Area adjacent to (c) showing Tlymphocyte (CD3-positive) aggregation around meningeal blood vessels. Meningeal lymphocytes are predominantly CD8-negative (e) and CD4-positive $(f)$. Lymphocytes within the cerebral neocortex are also predominantly CD8-negative $(g)$ and CD4-positive $(h)$. $\boldsymbol{i}$, Vacuolation and rarefaction of myelinated fibers in the cerebral white matter. $\boldsymbol{j}$, Infiltration of cerebral white matter by CD68-immunoreactive macrophages.
Removal and degradation of phagocytosed $A \beta$ occurred within $3 \mathrm{~d}$, as observed in vitro studies ${ }^{5}$ and by direct visualization in live immunized mice by multiphoton microscopy ${ }^{4}$. In our patient, despite periods of 20 months after the first immunization and 12 months after the last immunization, $\mathrm{A} \beta$ was still associated with microglia, indicating prolonged persistence of phagocytosed $A \beta$ or continuing phagocytosis.

The persistence of amyloid in the walls of blood vessels (CAA), despite its removal from plaques, was also observed in studies of PDAPP mice ${ }^{4}$. The vascular amyloid deposits, which comprise predominantly A 340 (unlike plaques, which are predominantly $\mathrm{A} \beta 42$ ), may be more stable, more rapidly replenished or less accessible, for example to A $\beta$-specific antibody or phagocytes ${ }^{4}$. A further possibility is that efflux of $A \beta$ from the brain through perivascular drainage pathways may be stimulated by the immunotherapy and contribute to $\mathrm{CAA}^{11}$. Whatever the mechanism, this relative persistence of vascular $A \beta$ may be relevant to the observation that CAA-related hemorrhage in APP transgenic mice was increased by one $A \beta$-specific antibody ${ }^{12}$.

Caution is required in extrapolating from the findings in this single case. There is considerable interindividual variation in the pathological features of $\mathrm{AD}$; some of the features described here might simply represent an unusual pattern of AD pathology, unrelated to the immunization. However, three features predicted by the mouse immunotherapy studies were identified in this patient immunized with $A \beta 42$. First, there were extensive areas with a low-density of $A \beta$ plaques without plaque-associated dystrophic neurites and GFAP-immunoreactive astrocytes. Second, $\mathrm{A} \beta$ immunoreactivity was associated with microglia in areas devoid of plaques. Third, there was persistence of cerebrovascular amyloid. On this basis, we favor the view that these observations represent therapeutic modification of the neuropathology of AD with removal of $A \beta$ from the human brain. Three additional features were not predicted by the mouse models of $A \beta$ immunotherapy: first, a $\mathrm{CD} 4^{+}$lymphocytic meningoencephalitis; second, persistence of neurofibrillary tangles and neuropil threads in areas devoid of plaques; and third, extensive macrophage infiltration of cerebral white matter.

Uncertainty remains over the consequences of removing $A \beta$ plaques from the brains of patients with established AD pathology. It is not known whether other features of AD pathology such as neuronal and synaptic loss will be affected, and whether cognitive improvements analogous to those seen in immunized PDAPP mice will occur. It also remains to be seen whether $A \beta$ immunotherapy given early in life could prevent accumulation of $\mathrm{A} \beta$ and, if so, whether other features of AD pathology such as those involving tau protein might also be prevented. Studies of $\mathrm{A} \beta$ immunotherapy are likely to provide a crucial test of the putative causal role of $\mathrm{A} \beta$ in the pathogenesis of $\mathrm{AD}$.

\section{Methods}

$A \beta$ antibody titers. Antibody titers were measured as previously described'; these data are available courtesy of D. Schenk (Elan Pharmaceuticals, South San Francisco, California). Briefly, A 342 was coated onto 96-well plates and incubated with various dilutions of patient serum in PBS. The amount of A $\beta$-specific antibody was ultimately detected with a horseradish peroxidase-linked second antibody against human IgG.

Neuropathology. All brains in this study were fixed in formalin and samples for histology were processed to paraffin wax by standard methods after macroscopic examination. Tissue from unimmunized AD cases satisfying CERAD criteria ${ }^{10}$ were drawn from the archives of the Neuropathology laboratory at Southampton General Hospital. The study received approval 
from the Southampton and South West Hants local research ethics committee. Standard methods were used for histological stains, including modified Bielschowsky, Congo red and thioflavine S. Immunohistochemistry was conducted using appropriate antigen retrieval methods for each antibody. We used primary antibodies against $A \beta$ (1:50; Novocastra, Newcastle, UK), Aß40 (1:250; Chemicon, Temecula, California), A $\beta 42$ (1:250; Chemicon), tau-2 (1:10,000; Sigma, Gillingham, UK), $\beta$-APP (1:100; Chemicon), human leukocyte antigen DR (CR3/43; 1:400; Dako, Glostrup, Denmark), IgG (1:1,000; Dako), IgM (1:1,000; Dako), C3 (1:1,000; Dako), CD3 (1:100; Novocastra), CD4 (1:10; Novocastra), CD8 (1:100; Novocastra), CD20 (1:400; Dako), CD45RO (1:50; Dako), CD68 (PGM1; 1:50; Dako) and CD79a (1:250; Dako). Bound primary antibody was visualized using a standard diaminobenzidine streptavidin-biotin horseradish peroxidase method (Dako).

Image analysis and quantification. $A \beta$ plaque immunoreactivity was assessed by computerized quantitative image analysis (Imaging Associates KS400 software, Bicester, UK) in the regions identified above. Ten $\times 10$ objective microscope fields (Zeiss Axioscop 2) were digitally captured (Zeiss Axiocam) from equivalent areas of each region from each case. Immunoreactivity is expressed as mean plaque density (plaques $/ \mathrm{mm}^{2}$ ) and $A \beta$ load (percentage immunostained area of region sampled). Tauimmunoreactive neurofibrillary tangles, tau-immunoreactive dystrophic neurite clusters and GFAP-immunoreactive astrocyte clusters were counted manually in $10 \times 10$ microscope fields by an experienced neuropathologist (J.N.). Neuropil threads were scored as $0=$ none, $1=$ sparse, $2=$ moderate and $3=$ dense. IgG immunoreactivity of plaques was scored as $1=$ faint staining of few plaques and 2 = faint staining of many plaques. Vascular $A \beta$ immunostaining (CAA) was scored according to published methods ${ }^{13}(0=$ none, $1=<1 / 3$ of vessels stained, $2=1 / 3$ to $2 / 3$ stained and $3=2 / 3$ to all stained)

\section{Acknowledgments}

We thank the family of the person whose details are described here for their permission to examine the brain and publish the findings; H.M. Coroner for Winchester for his permission to disclose this information; $D$. Schenk, $D$.
Games and others at Elan Pharmaceuticals for discussions and exchange of information; R. Alston and A. Page (Biomedical Imaging Unit, Southampton General Hospital) for help with image analysis and preparation of figures; and L. Murray for help with data presentation

\section{Competing interests statement}

The authors declare that they have no competing financial interests.

\section{RECEIVED 5 AUGUST 2002; ACCEPTED 14 FEBRUARY 2003}

1. Schenk, D. et al. Immunization with amyloid- $\beta$ attenuates Alzheimer-disease-like pathology in the PDAPP mouse. Nature 400, 173-177 (1999).

2. Janus, $C$. et al. A $\beta$ peptide immunization reduces behavioural impairment and plaques in a model of Alzheimer's disease. Nature 408, 979-982 (2000)

3. Morgan, D. et al. $\beta$ peptide vaccination prevents memory loss in an animal model of Alzheimer's disease. Nature 408, 982-985 (2000).

4. Bacskai, B.J. et al. Imaging of amyloid- $\beta$ deposits in brains of living mice permits direct observation of clearance of plaques with immunotherapy. Nat. Med. 7, 369-372 (2001)

5. Bard, F. et al. Peripherally administered antibodies against amyloid $\beta$-peptide enter the central nervous system and reduce pathology in a mouse model of Alzheime disease. Nat. Med. 6, 916-919 (2000).

6. Sigurdsson, E.M., Scholtzova, H., Mehta, P.D., Frangione, B. \& Wisniewski, T. Immunization with a non-toxic/nonfibrillar amyloid- $\beta$ homologous peptide reduces Alzheimer's disease-associated pathology in transgenic mice. Am. J. Pathol. 159, 439-447 (2001)

7. Birmingham, K. \& Frantz, S. Set back to Alzheimer vaccine studies. Nat. Med. 8 199-200 (2002).

8. Bishop, G.M., Robinson, S.R., Smith, M.A., Perry, G. \& Atwood, C.S. Call for Elan to publish Alzheimer's trial details. Nature 416, 677 (2002)

9. Check, E. Nerve inflammation halts trial for Alzheimer's drug. Nature 415, 462 (2002)

10. Ball, M. et al. Consensus recommendations for the postmortem diagnosis of Alzheimer's disease. Neurobiol. Aging 18, S1-S2 (1997).

11. Weller, R.O. et al. Cerebrovascular disease is a major factor in the failure of elimination of $A \beta$ from the aging human brain: implications for therapy of Alzheimer's disease. Ann. NY Acad. Sci. 977, 162-168 (2002)

12. Pfeifer M. et al. Cerebral hemorrhage after passive anti-A $\beta$ immunotherapy. Science 298, 1379 (2002)

13. McCarron, M.O. et al. The apolipoprotein E $\varepsilon 2$ allele and the pathological features in cerebral amyloid angiopathy-related hemorrhage. J. Neuropath. Exp. Neurol. 58, 711-718 (1999). 